\title{
A new approach to understanding the effects of surface wettability on nucleate boiling
}

\author{
Hai Trieu Phan ${ }^{1,2, a}$, Nadia Caney ${ }^{2}$, Philippe Marty ${ }^{2}$, Stéphane Colasson $^{1}$ \\ AND JÉRÔME GAVILLET ${ }^{3}$ \\ 1 LITEN/GRETh, CEA Grenoble, 17 rue des Martyrs, 38054 Grenoble Cedex 9, France \\ 2 LEGI, BP 53, 38041 Grenoble Cedex 9, France \\ ${ }^{3}$ LITEN/LTS, CEA Grenoble, 17 rue des Martyrs, 38054 Grenoble Cedex 9, France
}

Received 20 April 2009

\begin{abstract}
Although boiling process has been a major subject of research for several decades, its physics still remain unclear and require further investigation. This study aims at highlighting the effects of surface wettability on pool boiling heat transfer. Nanocoating techniques were used to vary the water contact angle from 20 to $110^{\circ}$ by modifying nanoscale surface topography and chemistry. The experimental results obtained disagree with the predictions of the classical models. A new approach of nucleation mechanism is established to clarify the nexus between the surface wettability and the nucleate boiling heat transfer. In this approach, we introduce the concept of macro- and micro- contact angles to explain the observed phenomenon.
\end{abstract}

Key words: Wettability / heat transfer / boiling / contact angle / nanocoating

Résumé - Une nouvelle approche pour comprendre les effets de la mouillabilité de surface sur l'ébullition nucléée. Cette étude a pour objectif d'étudier les effets de la mouillabilité sur l'ébullition en vase. L'angle de contact du fluide a été modifié par revêtement de nanoparticules sur des surfaces de test. Les résultats expérimentaux obtenus ne sont pas en accord avec les modèles classiques. Une nouvelle approche physique de l'influence de la mouillabilité sur l'ébullition en vase a été établie. Dans cette approche, nous introduisons les notions des macro- et micro-angles de contact afin d'expliquer les phénomènes observés.

Mots clés : Mouillabilité / transfert thermique / ébullition / angle de contact / nanostructuration

\section{Introduction}

Nucleate boiling is characterized by the liquid-vapour phase change associated with the bubble formation. It is an effective heat transfer mode which occupies an important place in engineering disciplines. Compared to singlephase process, this process enables to transfer more energy with a relatively lower temperature jump at the wall. During the last half of the twentieth century, significant advances were made in developing an understanding of the boiling heat transfer mode. Yet, because of the complexity of the process, the models and correlations developed often contain much simplification and could not explain recent observations. To explore the physics of the nucleate boiling process, further fundamental research is needed, especially on interfacial phenomena.

One of the major problems of the boiling field is the heterogeneous formation of bubbles from a wall. Several

\footnotetext{
${ }^{a}$ Corresponding author: hai-trieu.phan@cea.fr
}

researchers put forward the assumption that at the base of a bubble, there is an area where the three phases: solid, liquid and vapour are in contact. This area is called "triple contact line" $(T C L)$. Does the surface wettability, which is defined through the contact angle of a liquid droplet on the wall, have significant influence on the bubble formation mechanism? Up to now, this issue has not been fully resolved for lack of experimental data. The effects of the surface wettability on nucleate boiling parameters are usually explained by several classical models such as that of Fritz [1], Wang and Dhir [2] and Kandlikar [3]. Balancing the buoyancy force and the vertical component of the surface tension force, Fritz [1] determined the bubble departure diameter. He obtained a linear variation of the bubble departure diameter with the contact angle. Wang and Dhir [2] have conducted boiling experiments with copper heaters oxidized at different degrees in order to vary the contact angle. The authors observed that the increase of the surface wettability causes a decrease of the density of active nucleation sites. Analysing 


\section{Nomenclature}

\begin{tabular}{|ll|}
\hline$D_{\mathrm{d}}$ & bubble departure diameter, $\mathrm{m}$ \\
$f(\theta)$ & energy factor \\
$f_{\mathrm{e}}$ & bubble emission frequency, $\mathrm{s}^{-1}$ \\
$F_{\sigma}$ & surface tension force, $\mathrm{N}$ \\
$F_{\sigma_{-} \mathrm{h}}$ & horizontal component of $F_{\sigma}, \mathrm{N}$ \\
$F_{\sigma_{-} \mathrm{v}}$ & vertical component of $F_{\sigma}, \mathrm{N}$ \\
$g$ & gravity, $\mathrm{m} / \mathrm{s}^{2}$ \\
$h$ & heat transfer coefficient, $\mathrm{W} \cdot \mathrm{m}^{-2} \mathrm{~K}^{-1}$ \\
$N_{\mathrm{as}}$ & number of active nucleation sites \\
$t_{\mathrm{w}}$ & waiting time, $\mathrm{s}$ \\
$t_{\mathrm{g}}$ & growth time, $\mathrm{s}$ \\
& \\
Greek symbols \\
$\theta$ & contact angle, ${ }^{\circ}$ \\
$\theta^{\circ}$ & $\theta$ measured in air at $25^{\circ} \mathrm{C},{ }^{\circ}$ \\
$\theta^{s}$ & $\theta$ at saturated temperature, \\
$\theta_{\mu}^{s}$ & micro-contact angle, ${ }^{\circ}$ \\
$\rho$ & density, kg $/ \mathrm{m}^{3}$ \\
$\sigma$ & surface tension, $\mathrm{N} / \mathrm{m}$ \\
& \\
Subscripts & liquid \\
$\mathrm{l}$ & vapour \\
$\mathrm{v}$ & \\
Abbreviations \\
CHF & Critical heat flux \\
HTC & Heat transfer coefficient \\
TCL & triple contact line \\
\hline
\end{tabular}

the force resulting from the evaporation at the liquidvapour interface of a bubble, Kandlikar [3] demonstrated that the increase of the surface wettability induced the enhancement of critical heat flux $(C H F)$. Bao Truong et al. [4] combined these three models to show the mechanisms of the $C H F$ intensification and heat transfer coefficient deterioration, both attributed to the decrease of the contact angle. However, the scientific community always wondered about the existence of the $T C L$. As the latter causes the discontinuities of the physical quantities, some researchers, especially those working on numerical modelling, think that instead of the $T C L$, a continuous layer of non-evaporated liquid is present between the bubbles and the wall [5].

Up to now, few experimental data ara available to clarify the controversial understandings about the effects of the surface wettability on boiling heat transfer. Indeed, it is experimentally difficult to vary the contact angle while keeping all other parameters constant. A typical way to change the contact angle has been the use of surfactant solutions as described in Wen and Wang [6]. The disadvantage of this method is that both the surface wettability and the liquid surface tension vary simultaneously. Hence, the enhancement of the boiling heat transfer might be due to the significant decrease of the liquid surface tension rather than the increase of the surface wettability. Another way has been the surface treatment by microcoating such as the deposition of a microlayer of different materials on the heater surfaces [7-10]. However, this may disrupt the boiling process by changing the micro cavities density on the heater. Oxidizing copper [11] has been another method widely applied but this may also cause the change of the surface topography at microscale.

Today, the progresses in nanocoating allows modifying surface topography and chemistry at nanoscale. Particles of very small size (less than $100 \mathrm{~nm}$ ) named "nanoparticles" can be deposited on the heater surface. By changing the deposited particles material, we are able to vary the water contact angle within 0 and $180^{\circ}$. As the characteristic scale of the nucleation sites is micrometric, only the contact angle is changed while all other boiling parameters would remain constant. The techniques used in our laboratory are respectively MOCVD (Metal-Organic Chemical Vapour Deposition), PECVD (Plasma Enhanced Chemical Vapour Deposition) and NNBD (Nanofluids Nucleate Boiling Deposition). A pool boiling experiment was performed with the purpose of providing more experimental data to explore the influence of the surface wettability on the nucleate boiling mechanisms. A new physical approach of the nucleation is then established to understand the experimental results.

\section{Experimental apparatus}

The experimental setup is shown in Figure 1. The initial surface is made of a $20 \mu \mathrm{m}$ stainless steel (grade 301 ) foil which has a water contact angle of about $85^{\circ}$. It was cut to make seven sample heaters $100 \mathrm{~mm}$ long, $5 \mathrm{~mm}$ wide. One of them has been used as reference of the uncoated surface. The others were coated either by MOCVD, PECVD or NNBD to obtain different water contact angle from 20 to $110^{\circ}$ (Fig. 2). AFM (Atomic Force Microscope) scanning showed that in the initial state, the heater surfaces consist of parallel grooves $5 \mu \mathrm{m}$ wide and $100 \mathrm{~nm}$ deep. It is then expected that nanoparticles deposition with a thickness lower than $20 \mathrm{~nm}$ will not affect the surface microtopography. For each heater, the calibration of its electrical resistance as a function of the temperature was made beforehand. Hence, its temperature can be deduced from measurements of the electric resistance by using the electrical resistance/temperature curve. The sample heater was put in a boiling vessel containing pure water at $85^{\circ} \mathrm{C}$. The water temperature inside the vessel was maintained constant by a flow at very low flow rate from a thermostat. The sample heater was then heated by Joule effect to boil water near the heater surface. The experiment was done at atmospheric pressure. During the experiment, the boiling process was captured by a high speed camera with a recording speed of $6000 \mathrm{fps}$.

\section{Experimental results}

\subsection{Hydrophobic surfaces}

Figure 3 presents some captured images of the boiling process on the hydrophobic surfaces of which the static 


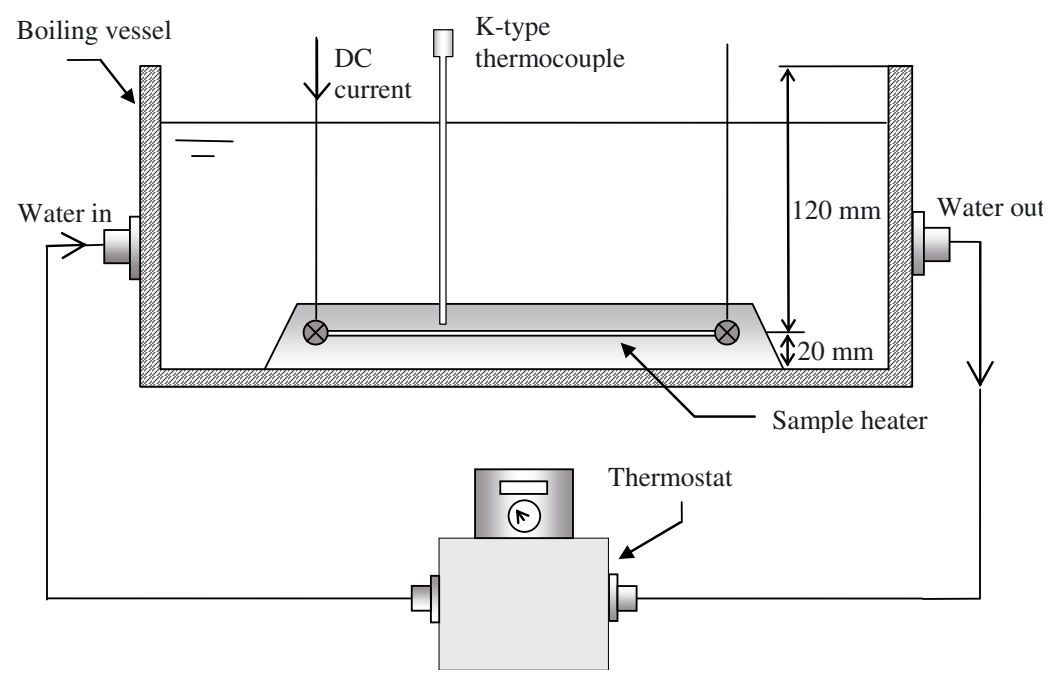

Fig. 1. Schematic view of the experimental setup.

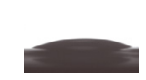

$22^{\circ}$

$\mathrm{SiOx}$

deposition

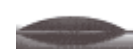

$31^{\circ}$

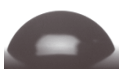

$67^{\circ}$

$\mathrm{TiO}_{2}$ deposition
$\mathrm{Pt}$

deposition

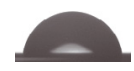

$80^{\circ}$

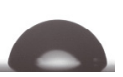

$85^{\circ}$

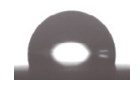

$104^{\circ}$

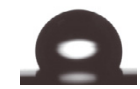

$112^{\circ}$

$\mathrm{Fe}_{2} \mathrm{O}_{3}$ deposition

Uncoated
$\mathrm{SiOC}$

deposition
Teflon

deposition

Fig. 2. Static contact angles of a $2-\mu \mathrm{L}$ sessile water-droplet on stainless steel surfaces with and without nanoparticles deposition (measured in air at $25^{\circ} \mathrm{C}$ ).

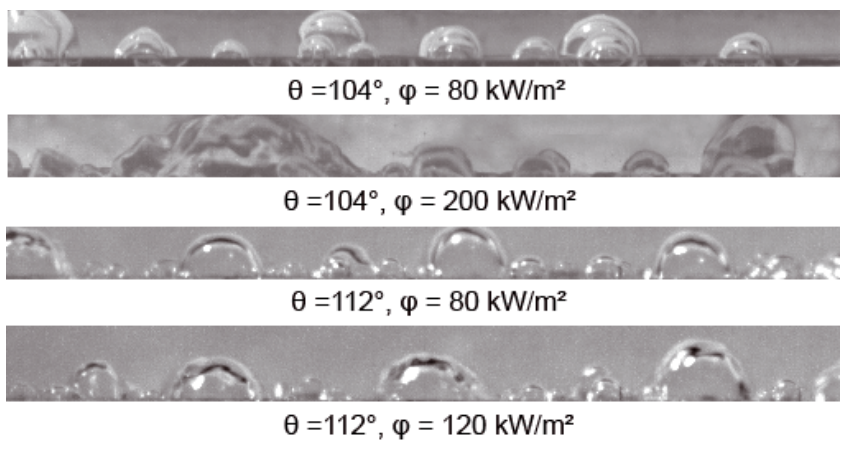

Fig. 3. For the hydrophobic surfaces, the bubbles appeared at very low superheats, but they could not detach from the wall. There was no nucleation and a film boiling configuration rapidly occurred because of the bubble coalescence.

contact angles at $25{ }^{\circ} \mathrm{C}$ are respectively 104 and $112^{\circ}$ (Fig. 2). Compared to the standard surfaces which are usually wetted, the bubbles appeared on the hydrophobic surfaces at a lower heat flux. Increasing the heat flux, the bubble size increased but the bubbles still did not detach from the wall. Actually, no bubble emission was observed on the hydrophobic surfaces. At higher heat flux, the bubbles spread over the surfaces, causing bubble coalescence that led to film boiling. These observations agree with these of Gaerther [8] and Hummel [9]: a continuous hydrophobic surface tends to become vapour blanketed during nucleate boiling. However, no bubble departure was noticed and the heat transfer was not stable when the bubbles stayed on the heater surfaces. The wall temperature increased with time and after about fifteen minutes, the local wall destruction occurred. Thus, it was impossible to measure the HTC in stationary regime.

\subsection{Hydrophilic surfaces}

By analysing the photos taken from the videos recorded by the high speed camera, we determined the bubble departure diameter $\left(D_{\mathrm{d}}\right)$ and the bubble emission frequency $\left(f_{\mathrm{e}}\right)$ for each hydrophilic surface. Figure 4 shows that a greater surface wettability yields bigger bubble departure diameter. This is in contrast with the correlation of Fritz [1] which estimates that $D_{\mathrm{b}}$ is proportional to the static contact angle. Further more, the duration of the bubble growth $\left(t_{\mathrm{g}}\right)$ and the time interval between the departure of each bubble and the appearance of the next bubble $\left(t_{\mathrm{w}}\right)$ were determined. It is logical to imagine that the formation of a bigger bubble requires a longer time. This is what we observed. Indeed, the decrease of the contact angle resulted in the increases of both $t_{\mathrm{g}}$ and $t_{\mathrm{w}}$. As a consequence, the bubble emission frequency, which is defined as $f_{\mathrm{e}}=1 /\left(t_{\mathrm{g}}+t_{\mathrm{w}}\right)$, decreases with the enhancement of the surface wettability (Fig. 5). Because of the uncertainties in the determination of $t_{\mathrm{g}}$ and $t_{\mathrm{w}}$ by high speed camera, the uncertainty of $f$ is relatively high when $f$ is greater than $200 \mathrm{~Hz}$.

The measurement of the density of active nucleation sites $\left(N_{\text {as }}\right)$ has been known to be difficult. To obtain reliable values, the heater surfaces must be uniform in terms 


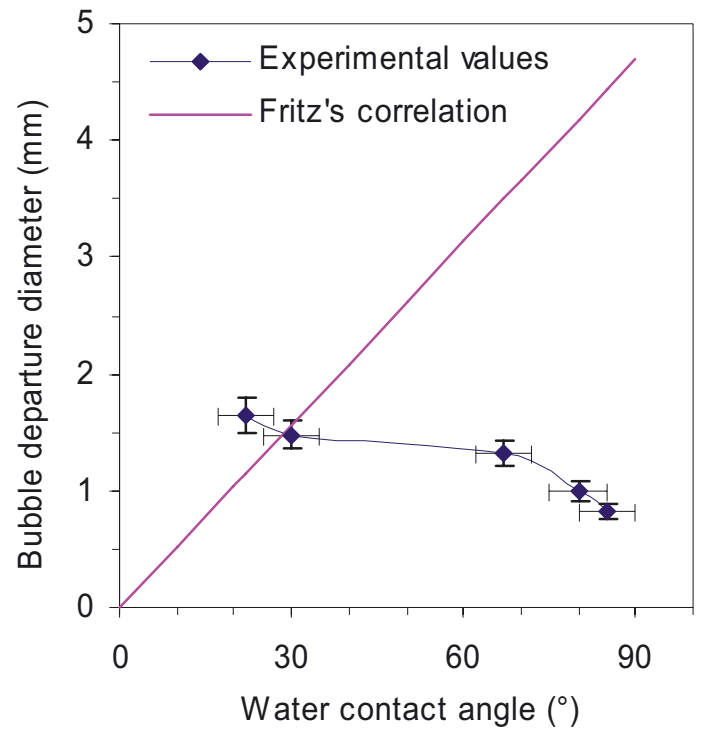

Fig. 4. Bubble departure diameter versus the contact angle measured at $25{ }^{\circ} \mathrm{C}$.

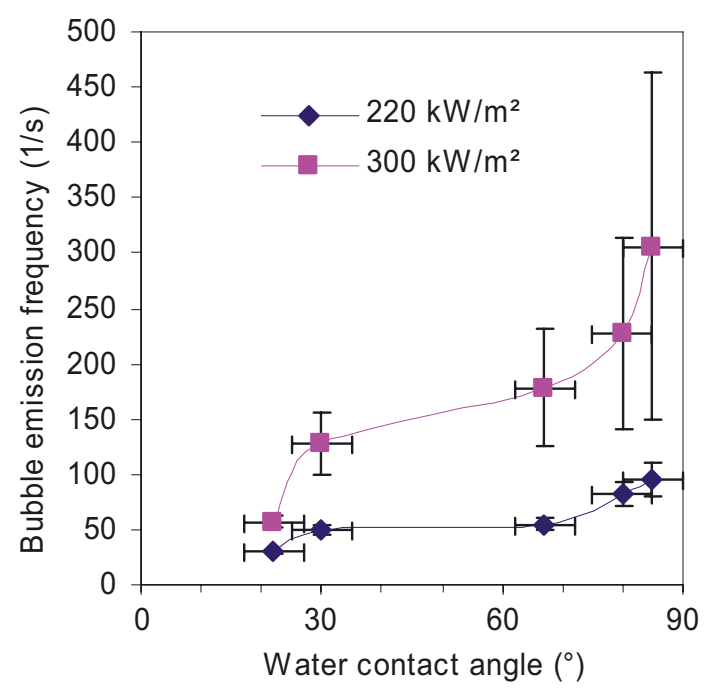

Fig. 5. Bubble emission frequency versus the contact angle measured at $25{ }^{\circ} \mathrm{C}$.

of the number and the size of the nucleation sites. On a large surface, this condition would never be satisfied. But the information about $N_{\text {as }}$ is useful to interpret the effects of the surface wettability on heat transfer. The high speed camera was positioned so that it provides a perspective view of the bubble generation. Then, $N_{\text {as }}$ was determined on a heater part $23 \mathrm{~mm}$ wide. We observed that at a given moment, $N_{\text {as }}$ decreased with the enhancement of the surface wetting. This is in agreement with what was described by Wang and Dhir [2]. However, when $N_{\text {as }}$ was averaged over a long period $(1 \mathrm{~s})$, it remained almost independent of the contact angle (Fig. 6). Figure 7 represents the variation of the heat transfer coefficient as a function of contact angle. It shows that $h$ deteriorates with the decrease of the contact angle when the latter is within 50 and $90^{\circ}$. Yet, when the contact angle is lower than $50^{\circ}$, its

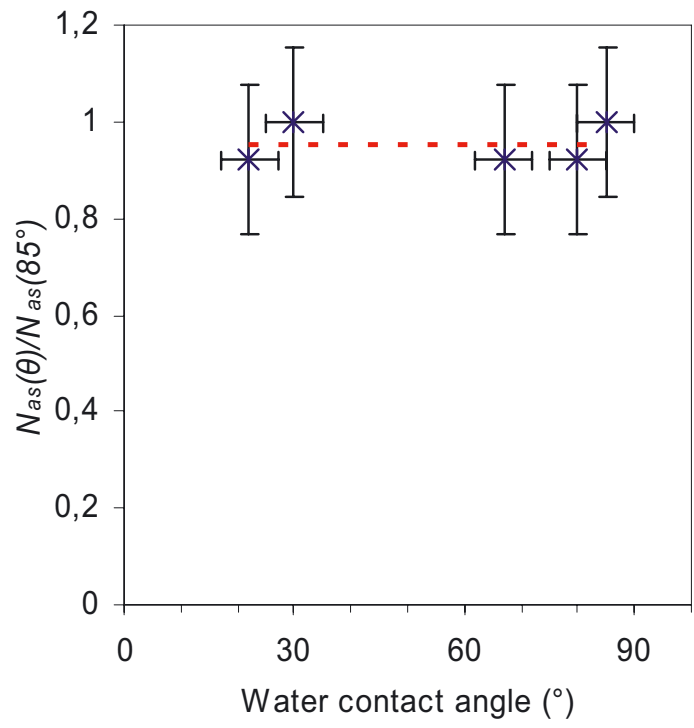

Fig. 6. Ratio of $N_{\text {as }}$ (averaged during $1 \mathrm{~s}$ on a heater part $23 \mathrm{~mm}$ wide) versus the contact angle measured at $25^{\circ} \mathrm{C}$.

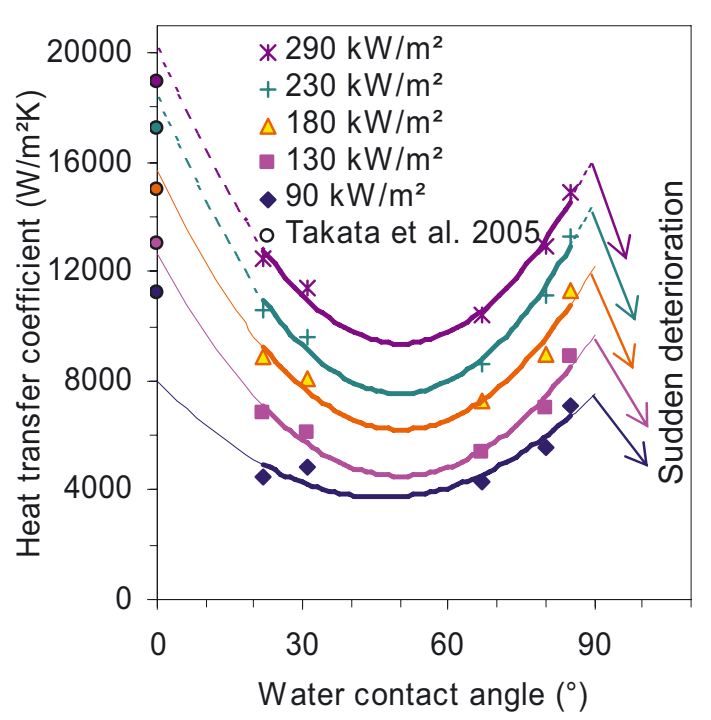

Fig. 7. Heat transfer coefficient versus the contact angle measured at $25{ }^{\circ} \mathrm{C}$.

decrease induces an increase of $h$. While working on superhydrophilic surfaces, Takata et al. [12] have observed the same phenomenon. These authors used dipping and sputtering techniques to deposit titanium dioxide $\left(\mathrm{TiO}_{2}\right)$ on smooth surfaces. Then, the coated surfaces were oxidized by ultraviolet radiation, creating the $\mathrm{TiO}_{2}$ photocatalysis that enables to obtain a water contact angle near $0^{\circ}$. For this angle, some points extracted from their experimental results are shown in Figure 7. They follow relatively well the trend of the curves fitting our results. Hence, the best heat transfer coefficient would be obtained with a surface of which the contact angle of is either 0 or $90^{\circ}$. Let us note that the experiment of Takata et al. [12] was performed with water at saturated temperature, whereas our experiment was conducted with subcooled water at 
$85^{\circ} \mathrm{C}$. But, it is well known that subcooling condition has a relatively weak influence on nucleate boiling.

\section{Physical approach}

This section presents our understanding of the influence of surface wettability on nucleate boiling mechanism.

\subsection{Existence of the triple contact line ( $T C L)$}

Our experimental results showed a significant influence of the surface wettability on the nucleated boiling. In particular, a clear difference of the bubble formation mechanism was observed between the hydrophobic and hydrophilic surfaces. Besides, the surface wettability affects the nucleation mainly through the contact angle resulting from the meeting of the three phases: solid, liquid and vapour. Therefore, it is reasonable to consider the $T C L$ in the processes of the bubble formation and growth.

\subsection{Macro- and micro-contact angles}

The contact angle is usually measured at room temperature $\left(25^{\circ} \mathrm{C}\right)$ by depositing a liquid droplet on the sample surface. The surface and the droplet are at the same temperature, thus there is no heat exchange between them. This contact angle is denoted as $\theta^{\circ}$. However, during the nucleate boiling, the bubble is formed by the liquid evaporation caused by the heat transfer from the wall to the liquid. The contact angle that follows $\left(\theta_{s}\right)$ is different from $\theta^{\circ}$, because the liquid is now at saturated temperature (Fig. 8). In general, the liquid surface tension decreases with the increase of the temperature. Hence, $\theta_{s}$ is lower than $\theta^{\circ}$ when the saturated temperature is higher than that of the room. In addition, at boiling conditions, the balance of the three surface energies: solid-liquid, liquid-vapour and solid-vapour, becomes unstable due to the non-zero heat flux imposed at the solid-liquid interface. For the hydrophilic surfaces, this heat flux causes the evaporation of the liquid microlayer underneath the bubble. The thinner this layer is, the higher heat flux passing through. Close to the $T C L$, the heat transfer would be extremely high and would create a liquid evaporation with a rate that is much higher than in the surrounding areas. Therefore, the curvature of the liquid-vapour interface would change, leading to the emergence of another contact angle named "microcontact angle" $\left(\theta_{\mu}^{s}\right)$. The contact angle $\theta_{s}$ is relatively at a larger scale. It is named "macro-contact angle".

\subsubsection{Influence of the micro-contact angle}

The surface tension force $\left(F_{\sigma}\right)$ is determined by the micro-contact angle and not by the macro-contact angle. When the nucleation is initiated, close to the $T C L$, the liquid evaporation may cause a micro-contact angle greater

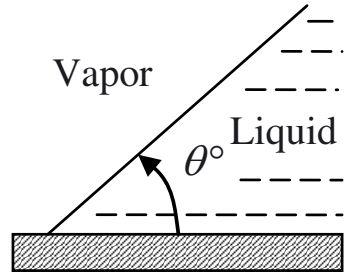

(a)

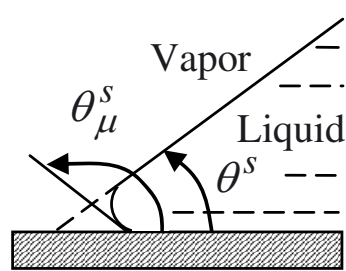

(b)
Fig. 8. (a) Contact angle of a liquid droplet at $25^{\circ}$ without any heat transfer; (b) contact angles of the bubble formed when the wall is heated: macro-contact angle $\theta^{s}$ and micro-contact angle $\theta_{\mu}^{s}$.

than $90^{\circ}$, as described by Mitrovic [13]. Due to the horizontal component of the surface tension force $\left(F_{\sigma-\mathrm{h}}\right)$, the liquid in the micro-layer moves backward from the bubble axis and the $T C L$ expands from A to B (Fig. 9). Along with the liquid movement, the micro-contact angle decreases as a result of the restoration of the surface energies balance. At position $\mathrm{B}$, the micro-contact angle is equal to $90^{\circ}$ and the surface tension force stops displacing the $T C L$. However, the liquid inertia and the energy minimization of the system will result in a decline of the micro-contact angle to a value close to that of the macrocontact angle. The horizontal component of the surface tension force reappears, but this time it moves the liquid forward by reducing the $T C L$ radius. At position $\mathrm{C}$, the $T C L$ disappears and the bubble detaches from the wall.

The micro-contact angle is an important parameter in nucleated boiling. First, it directly affects the vertical component of the surface tension force $\left(F_{\sigma-\mathrm{v}}\right)$, which contributes to maintain the bubble on the wall. Then, it creates the $T C L$ movement and thus affects the dynamic forces caused by the liquid inertia and viscosity. Indeed, when the $T C L$ is expanding from A to $\mathrm{B}$, the bubble becomes bigger and the inertia of the liquid surrounding the bubble exerts a reaction force to maintain it on the wall. But when the TCL retracts from $\mathrm{B}$ to $\mathrm{C}$, the liquid goes forward to the bubble axis, enabling the bubble departure. During the bubble growth, the macro-contact angle changes according to the hysteresis phenomenon: it decreases when the liquid recedes and increases when the liquid advances.

\subsubsection{Influence of the macro-contact angle}

Although the surface tension force depends on the micro-contact angle, the macro-contact angle always plays a key role. A simple way of understanding the effects of the macro-contact angle is to analyze its influence on the bubble form. The initial radius of the $T C L$ is assumed to be equal to that of the nucleation sites. For a simple geometry reason, the bubble which has a lower contact angle is bigger (Fig. 10). This might explain why the increase of the surface wettability leads to the increase of the energy needed to activate the nucleation sites. Therefore, 


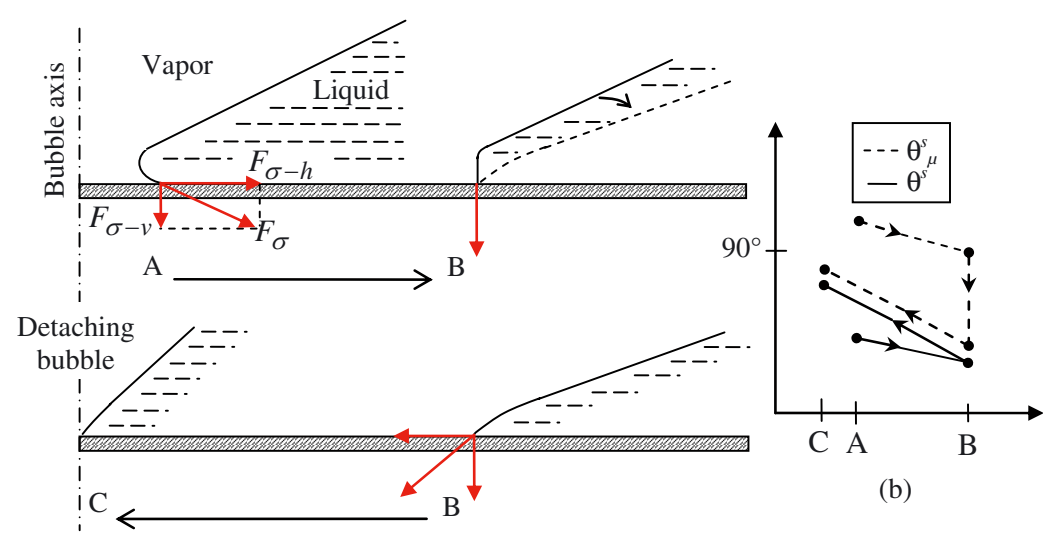

(a)

Fig. 9. (a) Nucleation initiation creates a micro-contact angle greater than $90^{\circ}$, causing the displacement of the $T C L$ from A to B. At position B, the horizontal component of the surface tension force becomes zero, but the liquid inertia and the energy minimisation of the system induces the decrease of the micro-contact angle to a value close to that of the macro-contact angle. Due to the surface tension force, the TCL retracts. Its radius tends to zero at C, enabling the bubble departure. (b) The change of the macro- and micro-contact angles during bubble growth.

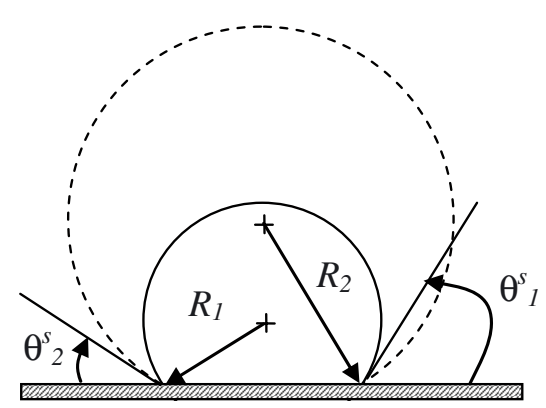

Fig. 10. Two spherical bubbles are assumed to have the same $T C L$. If $\theta_{2}^{s}$ is lower than $\theta_{1}^{s}$, the bubble of which the contact angle is $\theta_{2}^{s}$ is bigger than that having the contact angle $\theta_{1}^{s}$.

between the departure of a bubble and the appearance of the next one, the waiting time $\left(t_{\mathrm{w}}\right)$ would be longer when the macro-contact angle is lower. The decrease of the macro-contact angle would then intensify the phase difference caused by the time delay between the active nucleation sites (two nucleation sites are said to be "in phase" if they emit the bubbles at the same time). That might explain why, at a given time, while increasing the contact angle, we observed fewer active nucleation sites, whereas if the observation is made over a long period, $N_{\text {as }}$ remained almost independent of the macro-contact angle. We believe that the phase difference between the active nucleation sites is a major factor of the $C H F$ enhancement attributed to the increase of the surface wettability. Moreover, when the bubble is bigger, its growth time $\left(t_{\mathrm{g}}\right)$ increases. Hence, the increases of both $t_{\mathrm{w}}$ and $t_{\mathrm{g}}$ induce the decrease of the bubble emission frequency. Bankoff [14] defined the energy factor, $f(\theta)$, as the ratio of the energy needed to form a bubble with a contact angle $\theta$ to that needed to form a homogenous bubble with the same diameter. Its expression is given by:

$$
f(\theta)=\frac{2+3 \cos \theta-\cos ^{3} \theta}{4}
$$

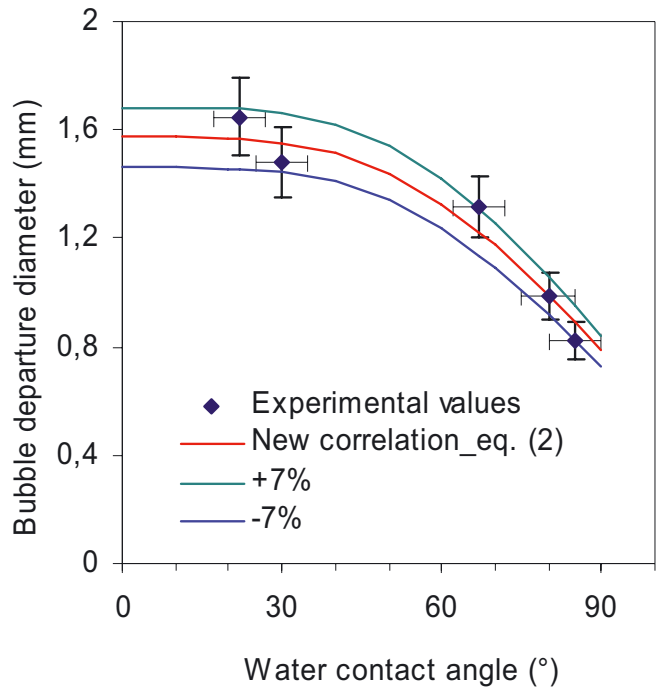

Fig. 11. New correlation to estimate the bubble departure diameter.

This factor is simply the volume ratio between a truncated sphere of which the contact angle is $\theta$ and a full sphere which has the same diameter. It could be representative for the influence of the macro-contact angle on the bubble form. Thereby, we established a new correlation of the bubble departure diameter which incorporates the influence of the fluid properties and the gravity as Fritz [1] did, but takes into account the energy factor as the contribution of the wetting effects:

$$
\begin{aligned}
D_{d}= & 0,626977 \frac{\left(2+3 \cos \theta-\cos ^{3} \theta\right)}{4} \\
& \times\left(\frac{\sigma}{g\left(\rho_{l}-\rho_{v}\right)}\right)^{1 / 2}
\end{aligned}
$$

Figure 11 shows that this new correlation fits relatively well the experimental results with only $7 \%$ difference. 


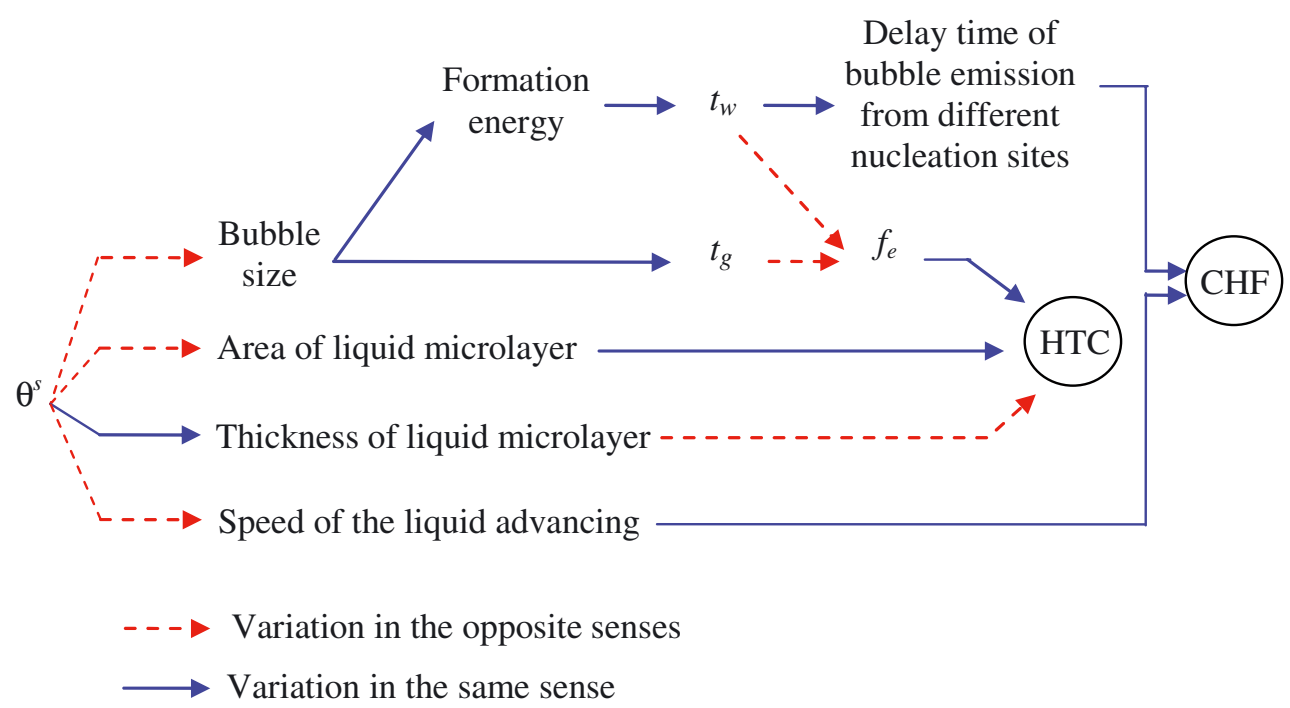

Fig. 12. Logical diagram describing the effects of the macro-contact angle on the nucleated boiling parameters.

Furthermore, the macro-contact angle affects the heat transfer through its effect on the liquid micro-layer underneath the bubble. For a lower macro-contact angle, this area would be thinner and larger, improving $h$. Thus, the results shown in Figure 7 could be explained by analyzing the compromise between the effect on $f_{\mathrm{e}}$ and that on the liquid micro-layer. At $90^{\circ}$ macro-contact angle, there is an optimum of $h$ as $f_{\mathrm{e}}$ is maximum. When the macro-contact angle is within 50 and $90^{\circ}$, the effect on $f_{\mathrm{e}}$ dominates and the decrease of $f_{\mathrm{e}}$ (due to the decrease of the macro-contact angle) causes the deterioration of $h$. However, when the macro-contact angle is lower than $50^{\circ}$, the effect on the liquid micro-layer dominates. Despite the fact that $f_{\mathrm{e}}$ is the lowest, at $0^{\circ}$ macro-contact angle, the bubbles stay longer time on the heater surface with the largest and thinnest liquid micro-layer, inducing another optimum of $h$.

Through its influence on the liquid micro-layer, the macro-contact angle also affects the micro-contact angle and especially on the movements of the $T C L$. For a lower macro-contact angle, the expansion of the $T C L$ would be longer but its retraction would be faster. We believe that the speed of the liquid advancing when the $T C L$ goes from B to C (Fig. 9) plays an important role in the $\mathrm{CHF}$ enhancement mechanism. Actually, the $C H F$ would be improved if this speed becomes higher, for example by increasing the capillary length of the coating. This might explain why at the same contact angle, Kim et al. [15] observed a better $\mathrm{CHF}$ for the more porous coating.

Figure 12 summarizes the effects of the macro-contact angle on the nucleated boiling.

\section{Conclusion}

Our experimental results showed that the surface wettability has significant effects on the nucleated boiling.
They tend to prove the existence of the triple contact line at the base of the bubbles. Near this line, because of the ultra-high rate of liquid evaporation, the curvature of the liquid-vapour interface changes, causing a change of the contact angle. Thus, we distinguish micro- and macro-contact angles. Due to the micro-contact angle, the $T C L$ expands and then retracts during the bubble growth, creating the liquid movements that are responsible for the macro-contact angle hysteresis and the bubble departure. The influence of the macro-angle on the bubble form has been analysed to explain the experimental observations, especially those concerning the heat transfer coefficient and the critical heat flux.

\section{References}

[1] W. Fritz, Maximum volume of vapor bubbles, Phys. Z. 36 (1935) 379-384

[2] C.H. Wang, V.K. Dhir, Effect of surface wettability on active nucleate site density during pool boiling of water on a vertical surface, Trans ASME J. Heat Transfer 115 (1993) 670-679

[3] S.G. Kandlikar, A theoretical model to predict pool boiling FC incorporating effects of contact angle and orientation, J. Heat Transfer 123 (2001) 1071-1079

[4] B. Truong, L.W. Hu, J. Buongiorno, Surface modifications using nanofluids for nucleate boiling heat transfer, ICNMM2008-62085, Darmstadt, Germany, 2008

[5] Y.A. Buyevich, B.W. Webbon, Dynamics of vapour bubbles in nucleate boiling, Int. J. Heat Mass Transfer 39 (1996) 2409-2426

[6] D.S. Wen, B.X. Wang, Effects of surface wettability on nucleate pool boiling heat transfer for surfactant solutions, Int. J. Heat and Mass Transfer, 45 (2002) 1739-1747

[7] P. Griffith, J.D. Wallis, The role of surface conditions in nucleate boiling, Chem. Eng. Prog. Symp. Ser. 56 (1960) 49-63 
[8] R.F. Gaertner, Methods and means for increasing the heat transfer coefficient between a wall and boiling liquid, US Patent 3 (1967) 301-314

[9] R.L. Hummel, Means for increasing the heat transfer coefficient between a wall and boiling liquid, US Patent 3 (1965) 207-209

[10] R.I. Vachon, G.H. Nix, G.E. Tanger, Evaluation of constants for the Rohsenow pool boiling correlation, J. Heat Trans. 90 (1968) 239-247

[11] S.C. Johnathan, J. Kim, Nanofluid boiling: The effect of surface wettability, Int. J. Heat and Mass Transfer, doi:10.1016/j.ijheatfluidflow.2008.07.004
[12] Y. Takata, S. Hidaka, J.M. Cao, T. Nakamura, H. Yamamoto, M. Masuda, T. Ito, Effect of surface wettability on boiling and evaporation, Energy 30 (2005) 209-220

[13] J. Mitrovic, Formation of a liquid jet after detachment of a vapour bubble, Int. J. Heat and Mass Transfer 40 (1997) 4309-4317

[14] S.G. Bankoff, Ebullition from solid surfaces in the absence of a pre-existing gaseous phase, Trans. Am. Mech. Engrs. 79 (1957) 735-740

[15] H. Kim, M. Kim, Effect of nanoparticle deposition on capillary wicking that influences the critical heat flux in nanofluids, Appl. Phys. Lett. 91 (2007) 014104 